\title{
Evaluación de factores de riesgo que afectan la mortalidad en pollos de engorde durante el proceso de traslado granja-planta de faenamiento en el centro norte de la región interandina
}

\section{Evaluation of risk factors affecting mortality in broiler chickens during the farm-laughterhouse transfer process in the northern center of the inter-Andine region}

\author{
Carlos Patricio Torres-Vinueza ${ }^{1}$, Lenin Javier Ron-Garrido², Jorge Eduardo Grijalva-Olmedo ${ }^{3 *}$ \\ ${ }^{1}$ Universidad Central del Ecuador, Facultad de Medicina Veterinaria y Zootecnia. Jerónimo Leiton y Gato Sobral, Quito, Ecuador. \\ 凶 cptorres@uce.edu.ec \\ https://orcid.org/0000-0001-8302-833X \\ ${ }^{2}$ Universidad Central del Ecuador, Facultad de Medicina Veterinaria y Zootecnia. Jerónimo Leiton y Gato Sobral, Quito, Ecuador. \\ \1jron@uce.edu.ec \\ https://orcid.org/0000-0001-9021-4376 \\ ${ }^{3}$ Universidad Central del Ecuador, Facultad de Medicina Veterinaria y Zootecnia. Jerónimo Leiton y Gato Sobral, Quito, Ecuador. \\ jgrijalva@uce.edu.ec \\ https://orcid.org/0000-0001-8301-531X
}

\begin{abstract}
Resumen
Millones de pollos de engorde son transportados anualmente desde las granjas hasta las plantas de faenamiento. En este trayecto, los pollos están expuestos a una amplia variedad de factores estresantes como la restricción de alimento, la captura y el transporte que afectan el bienestar animal y producen pérdidas de peso vivo hasta la muerte, lo cual impacta económicamente la actividad. El objetivo del presente estudio fue evaluar los factores de riesgo que afectan la mortalidad de pollos de engorde durante su transporte a la planta de faenamiento, a partir de observaciones y análisis de una muestra de 60 camiones con varios destinos del país. La incidencia de mortalidad promedio en el estudio fue de 1,6 por cada 1.000 pollos, cuyas variables de mayor impacto sobre la mortalidad durante el transporte fueron, la temperatura de embarque al causar un incremento del 3,4\% de mortalidad por cada $1{ }^{\circ} \mathrm{C}$ de descenso en la temperatura respecto de la mortalidad base; y, la edad de los pollos al momento de embarque que causó un incremento de $17 \%$ en la mortalidad por cada día adicional de estancia en finca, en relación con la tasa de mortalidad base. En conclusión, la mortalidad durante el faenado se incrementó en los pollos de engorde de mayor edad, así como por la disminución de la temperatura al momento del embarque. Estos factores de riesgo identificados pueden utilizarse para perfeccionar las prácticas de gestión a fin de mitigar parte del riesgo de mortalidad de pollos de engorde durante la transportación.
\end{abstract}

Palabras clave: bienestar animal, transporte, temperatura ambiente, estrés térmico.

\begin{abstract}
Millions of broiler chickens are transported annually from farms to the laughterhouse along the way, chickens are exposed to a wide variety of stressors such as food restriction, capture and transport, which affect animal welfare and cause live weight losses to death, which economically impacts activity. The objective of this study was to assess risk factors affecting fattening chicken mortality during transport to the laughterhouse, based on observations and analysis of a sample of 60 trucks with various destinations in the country. The average morta-
\end{abstract}


lity rate in the study was 1.6 per 1000 chickens, whose highest impact variables on mortality during transport were the boarding temperature, causing a $3.4 \%$ increase in mortality per $1{ }^{\circ} \mathrm{C}$ in temperature compared to base mortality; and, the age of the chickens at the time of shipment that caused a $17 \%$ increase in mortality for each additional day of stay on the farm, relative to the base mortality rate. In conclusion, mortality during fishing was increased in older broiler chickens, as well as by decreased temperature at the time of shipment. These identified risk factors can be used to refine management practices to mitigate some of the risk of fattening chicken mortality during transportation.

Keywords: Animal welfare, transportation, ambient temperature, thermal stress

\section{Introducción}

En la actualidad, la industria avícola ecuatoriana constituye una cadena productiva de gran escala. Diariamente se transportan millones de pollos de engorde desde las granjas donde se crían hasta las plantas de faenamiento, donde finalmente son procesados. Las estadísticas del país indican un crecimiento en la producción de 220 a 230 millones de aves de engorde en un lapso de cinco años desde 2012 a 2016 (Ruiz, 2017). Así mismo, el consumo per cápita de carne de pollo actualmente supera $\operatorname{los} 30-32 \mathrm{~kg}$ anuales, lo cual explica una alta producción y volúmenes de animales trasportados (Corporacion Nacional de Avicultores del Ecuador [Conave], 2021).

El transporte de pollos es un proceso que involucra diversas actividades, entre ellas: embarque en la granja, transporte hasta el sitio de faenado y distribución. Según diversos estudios epidemiológicos, son varios los factores de riesgo implicados en la salud y bienestar animal, incluyendo la línea genética, el tamaño del lote, el peso vivo, la tasa de mortalidad durante el período de cría, el método de captura, la hora del día, densidad de carga de las cajas donde se alojan los pollos, las condiciones climáticas durante el viaje y la duración de la carga (Nijdam et al., 2004; Whiting et al., 2007; Caffrey et al., 2017; Teke, 2019; Arbor Acres, 2019; Cuadros Colmenares, 2006; Vecerkova et al., 2019; Villarroel et al., 2018). La tasa de mortalidad al momento del arribo de los camiones al sito de entrega se estima en $1,63 \%$, con un rango que oscila entre $1,56 \%$ en ayuno de alimento de menos de 12 horas y $1,69 \%$ en los pollos que carecen de agua por más de 8 horas (Prado et al., 2010).

Durante la fase final del ciclo de producción, el manejo incluye el ayuno, captura y transporte de pollos; lo más traumático es la recolección de éstos, los cambios en la temperatura y la humedad relativa, así como las condiciones de las vías (Moreno y Chinchi1la, 2007, Villarroel et al., 2018). Estos factores que ocurren durante el proceso de transporte determinan que los pollos se expongan a cambios que provocan estrés y pierdan peso (Bayliss \& Hinton, 1990). La incidencia de mortalidad que registran los pollos de engorde durante el transporte y manipulación antes del sacrificio, conduce finalmente a una pérdida económica para la industria avícola y un problema de bienestar animal (Warriss, 2005).

En la planta de faenamiento, cuando los pollos de engorde se descargan de sus cajas, las aves muertas se describen como "muertas a la llegada" o DOA (Dead on Arrival, por sus siglas en inglés). Sin embargo, la muerte pudo ocurrir en cualquier momento después de la carga en granja, o durante el viaje. Como esos pollos muertos son considerados no aptos para el consumo humano, representan una pérdida económica. El riesgo de mortalidad o el porcentaje de DOA en una carga de aves proporciona una indicación de la gravedad de los problemas de bienestar animal que experimentan los pollos durante el transporte (Jacobs et al., 2017). La variabilidad en las tasas de DOA entre cargas, plantas de matanza y aún entre países, sugiere la existencia de múltiples factores de riesgo de mortalidad. Si se logran establecer estos factores de riesgo, las interrelaciones y su contribución relativa, sería posible identificar prácticas de gestión que reduzcan el riesgo de mortalidad (Nijdam et al., 2004; Bulent, 2019; Vecerkova et al., 2019).

Bayliss y Hinton (1990) sugieren que la influencia de algunos de esos factores múltiples sobre el riesgo de mortalidad podría relacionarse con el estado de salud del lote, ya que los pollos pueden estar sufriendo de una enfermedad terminal o ser más vulnerables a extremos ambientales. Al respecto, Mitchell y Kettlewell (2009) sostienen que esos factores pueden aparecer por afecciones, como insuficiencia cardíaca congestiva y ascitis. 
Otro factor de riesgo según Whiting et al. (2007) y Knezacek et al. (2010) parece ser el estrés térmico que ocurre en condiciones ambientales extremas durante la carga, el transporte y estabulación. De igual forma, un factor adicional ocurre por lesiones físicas antes del transporte, durante la captura y la carga. Es probable que las proporciones relativas de pollos que mueren a causa de estos factores varíen según las condiciones ambientales que experimentan durante el viaje. Cuando las características del viaje son tales que exceden la capacidad termorreguladora de los pollos, u homeotermia, aumenta el riesgo de mortalidad por la carga y crece el porcentaje de aves que mueren debido al estrés térmico (Hunter et al., 2001; Burlinguette et al., 2012; Knezacek et al., 2010; Habibu et al., 2014). Los pollos pueden morir de hipertermia si las condiciones son demasiado cálidas y húmedas, $\mathrm{o}$ de hipotermia si las condiciones son demasiado frías y los pollos están húmedos y fríos (Warriss et al. 2005). Además, es probable que se reduzca la calidad de la carne de los pollos que sobreviven a los viajes difíciles (Dadgar et al. 2011; Mitchell y Kettlewell, 1998; Weeks, 2012; Barbut, 2015).

Los pollos bajo estrés durante el transporte, consumen reservas de glucógeno muscular que provoca cambios en el pH final de la carne. Los cambios de $\mathrm{pH}$ de la carne postmortem, producen cambios estructurales en las fibras musculares, afectando la capacidad de retención de agua en la carne y la sensibilidad. La intensidad y duración del estrés del pollo provoca que la carne tome ciertas características: carne pálida, suave, exudativa (PSE, por sus siglas en inglés) y en otras ocasiones carne oscura, firme y seca (DFD, por sus siglas en inglés). Según varios autores, el estrés en un período corto previo al sacrificio, resulta en carne PSE, y durante períodos largos de estrés una carne con características DFD (Adzitey y Nurul, 2011; Webster et al., 1992).

Por lo expuesto, el transporte es el escenario con mayor impacto sobre el bienestar animal, ya que durante el transporte los pollos de abasto están expuestos a factores de estrés que incluyen microclimas de transporte que comprometen el confort térmico, lo cual puede dar lugar al deterioro de la calidad y el rendimiento de la canal, con cambios visibles en el color, olor y textura del producto final (Dadgar et al., 2011; Grill et al., 2013).

Con estos antecedentes, se planteó la presente investigación con el propósito de evaluar los factores de riesgo asociados a la mortalidad de pollos de engorde durante el proceso de transporte desde la granja de producción hacia la planta de faenamiento, en la zona centro norte de la región interandina del Ecuador. Esta información contribuirá a determinar si el porcentaje de mortalidad que se presenta en los pollos durante el transporte está relacionado con las variables ambientales y/o manejo que se da en una granja de producción.

\section{Materiales y métodos}

\subsection{Descripción del sitio de estudio}

El estudio se llevó a cabo en la granja Proviacea Cía. Ltda., ubicada en la provincia de Pichincha, Ecuador, cuyas coordenadas geográficas son: UTM $9981560 \mathrm{~N}, 778280 \mathrm{E}$, a una altitud de 2.000 m s.n.m., temperatura promedio de $20{ }^{\circ} \mathrm{C}$ y $60 \mathrm{~mm}$ mensuales de precipitación. De igual forma, se siguieron varias rutas de transporte de pollos hacia diferentes destinos situados mayoritariamente en el centro-norte de la región interandina, en altitudes comprendidas entre 2.000 y $3.000 \mathrm{~m}$ s.n.m. y un amplio rango de 08 a $25{ }^{\circ} \mathrm{C}$ de temperatura, en sitios de desembarque.

\subsection{Diseño del estudio y colección de datos}

Se realizó un estudio transversal observacional en una población constituida por 900 mil pollos de engorde de las líneas genéticas Cobb 500 y Ross 308. Los pollos se diferenciaron en machos y hembras mediante observación de sus características fenotípicas. La edad y peso vivo promedio de los pollos al momento del sacrificio fue de $50 \pm 1,125$ días y $2,7 \mathrm{~kg}$, respectivamente, cuyos datos fueron tomados luego de terminar el proceso de crianza en galpones con ventilación controlada de la granja de producción avícola. En el sitio de embarque en granja, se contabilizó el número de pollos embarcados vivos por camión.

Los camiones eran unidades abiertas lateralmente, con capacidad de 40 cajas o jaulas que pueden albergar hasta 8 pollos cada una. En cada uno de los 60 camiones, se realizó un muestreo aleatorio de 5 jaulas, las cuales fueron identificadas con una cinta o brocheta. En cada jaula se registraron datos de mortalidad de pollos, así como el número de pollos embarcados. Se registró la temperatura de embarque y de desembarque utilizando un termómetro en ${ }^{\circ} \mathrm{C}$ y la humedad relativa en $\%$ utilizando un higrómetro tanto al inicio del embarque como en el sitio de destino. Se registró la distancia recorrida en $\mathrm{km}$ y la velocidad prome- 
dio del transporte durante el viaje en $\mathrm{km} \mathrm{hora}^{-1}$, así como el estado de las vías en primer, segundo o tercer orden.

Una vez en la planta de faenamiento, se registró el número de pollos muertos, expresado en \% del total de pollos transportados. De igual forma, se registró probables anomalías externas visibles, tales como: laceraciones, heridas, cortes y contusiones.

\subsection{Análisis de datos}

Se utilizó estadística descriptiva para detallar las variables tiempo de ayuno, factores de carga y transporte, peso de los pollos, temperatura y humedad relativa ambiental. Los datos fueron analizados mediante un modelo lineal generalizado $(\mathrm{glm})$ utilizando el software estadístico R (v.3.6.3) en plataforma RStudio (Version 1.2.5033). El grado de asociación existente entre las covariables tiempo, línea genética, sexo, peso, se realizó por medio de un modelo de regresión Poisson, de acuerdo con el modelo estadístico [1].

$$
\begin{gathered}
\log \left(\lambda_{i}\right)=\log n_{i}+\sum X_{i} \beta_{i} \\
E\left(Y_{i}\right)=\lambda_{i}
\end{gathered}
$$

donde:

- $\quad Y_{i}$ : representa el valor de la variable respuesta para la observación i-ésima (número de pollos al i-ésimo camión)

- $\quad \lambda_{i}$ : Tasa de número de casos en alguna categoría o combinación de variables.

- $\quad X_{i}$ : Valor de la variable explicativa para la observación i-ésima

- $\quad \beta_{j}$ : Coeficiente de regresión para cada una de las $X \mathrm{~s}$ variables

- $E(Y)$ : Esperanza de la variable $Y$, misma que está representada por la tasa de mortalidad.

- $\log \left(n_{i}\right)$ : Logaritmo del número de pollos embarcados.

Las variables utilizadas en el estudio se describen en la Tabla 1.

Tabla 1. Promedios y desviación estándar de las variables analizadas en el estudio

\begin{tabular}{lcc}
\multicolumn{1}{c}{ Variable } & Promedio & Desviación estándar \\
\hline Tiempo de viaje, horas & 2,67 & 1,56 \\
Distancia recorrida, km & 123,28 & 92,91 \\
Velocidad media en el viaje, km/hora & 44,21 & 12,63 \\
Temperatura de embarque, ${ }^{\circ} \mathrm{C}$ & 21,25 & 3,67 \\
Temperatura de desembarque, ${ }^{\circ} \mathrm{C}$ & 13,58 & 6,41 \\
Humedad relativa, $\%$ & 83,65 & 10,45 \\
Edad de los pollos, días & 50,00 & 1,13 \\
Capacidad del camión, $\mathrm{N}^{\circ}$ de pollos & 326,73 & 51,00 \\
\hline
\end{tabular}

Adicionalmente, se usó el procedimiento Stepwise para la selección de modelos y de variables (factores de riesgo). La definición del mejor modelo

\section{Resultados}

La Tabla 2 describe la relación entre las variables estudiadas con la mortalidad de pollos. La mortalidad registrada fue de 1,6 por cada 1.000 po- de ajuste se realizó utilizando el Criterio de Información de Akaike (AIC) donde el valor más bajo se consideró como mejor ajuste.

llos $\left(\mathrm{IC}_{95 \%}: 1,4\right.$ a 1,9 por cada 1.000 pollos), que representan 213 pollos muertos durante la movilización de 130.567 pollos embarcados en granja hasta su arribo a la planta de faenamiento en los 60 camiones evaluados. 
Tabla 2. Coeficientes del modelo de regresión de Poisson de los factores de riesgo de mortalidad de pollos de engorde durante el transporte a la planta de faenamiento.

\begin{tabular}{|c|c|c|c|c|}
\hline Coeficientes & Estimador lineal & Error estándar & $z$ value & $\mathbf{P}(>|\mathbf{z}|)$ \\
\hline (Intercept) & $-14,0800$ & 5,3840 & $-2,615$ & 0,00892 \\
\hline Edad & 0,1937 & 0,0957 & 2,024 & 0,04301 \\
\hline Sexo aves machos & $-0,1254$ & 0,2650 & $-0,473$ & 0,63602 \\
\hline Temperatura embarque & $-0,0508$ & 0,0249 & $-2,041$ & 0,0413 \\
\hline Capacidad de jaulas & $-0,0008$ & 0,0016 & $-0,477$ & 0,63357 \\
\hline Raza aves Ross & $-0,3183$ & 0,4925 & $-0,646$ & 0,51806 \\
\hline Tiempo de viaje & $-0,1581$ & 0,2583 & $-0,612$ & 0,54047 \\
\hline $\mathrm{Km}$ recorridos & 0,0058 & 0,0056 & 1,023 & 0,30646 \\
\hline Velocidad & $-0,0165$ & 0,0180 & $-0,916$ & 0,35987 \\
\hline Temperatura de desembarque & $-0,0274$ & 0,0182 & $-1,505$ & 0,13245 \\
\hline HR al desembarque & 0,0005 & 0,0093 & 0,053 & 0,9574 \\
\hline
\end{tabular}

Las variables: sexo de las aves, capacidad de las jaulas (326,73 \pm 51 aves), raza (Cobb vs. Ross), tiempo de viaje $(2,67 \pm 1,56$ horas), kilómetros recorridos $(123,28 \pm 92,91 \mathrm{~km})$, velocidad media de viaje $(44,21 \pm 12,63 \mathrm{~km} /$ hora $)$ y humedad relativa $(83,65 \pm 10,45 \%)$ no presentaron asociación con la tasa de mortalidad. Por el contrario, tanto la temperatura de embarque $\left(21,25 \pm 3,67{ }^{\circ} \mathrm{C}\right)$ como la temperatura de desembarque $\left(13,58 \pm 6,41{ }^{\circ} \mathrm{C}\right)$ tuvieron relación con la mortalidad de pollos. En este último caso, la relación fue de tipo negativa al demostrarse que el descenso de un grado en la temperatura de embarque, incrementa la mortalidad en $5,2 \%$ de pollos. Igualmente, las estimaciones de mortalidad de pollos por efecto de la temperatura de desembarque, resultó en un incremento del 2,8\% en la mortalidad por cada $1{ }^{\circ} \mathrm{C}$ de descenso en la temperatura al desembarque.

Por otra parte, y como variables intrínsecas, la edad de los pollos acusó una correlación positiva con la mortalidad durante el transporte, esto es, los pollos con más días que el promedio de edad, acusaron un $17 \%$ más de mortalidad por cada día de permanencia adicional en galpón.

En la Tabla 3 se puede apreciar el modelo reducido de la mortalidad en los pollos, que explica el efecto de la edad de las aves y la temperatura de embarque sobre la mortalidad de los pollos. La edad presentó alta significación con el incremento en la tasa de mortalidad en relación con la temperatura de embarque.

Tabla 3. Coeficientes del modelo de regresión de Poisson de la mortalidad de pollos debido al efecto de la edad de los pollos y temperatura de embarque.

\begin{tabular}{|c|c|c|c|c|}
\hline Coeficientes & Estimador lineal & Error estándar & $\mathrm{z}$ value & $\mathbf{P}(>|\mathbf{z}|)$ \\
\hline (Intercept) & $-13,51453$ & 3,37343 & $-4,006$ & 0,000 \\
\hline Edad & 0,15549 & 0,06620 & 2,349 & 0,0188 \\
\hline Temperatura de embarque & $-0,03559$ & 0,01812 & $-1,964$ & 0,0495 \\
\hline
\end{tabular}

De esta forma, la edad de los pollos al momento de la captura se relacionó con una mortalidad extra del 17\% (IC ${ }_{95 \%}: 2,6-33,0 \%$ ) respecto de la mortalidad base por cada día de edad adi- cional que se mantuvieron en galpón. En la Figura 1 se puede apreciar la relación positiva entre la edad de los pollos y el log (aves muertas/aves embarcadas). 


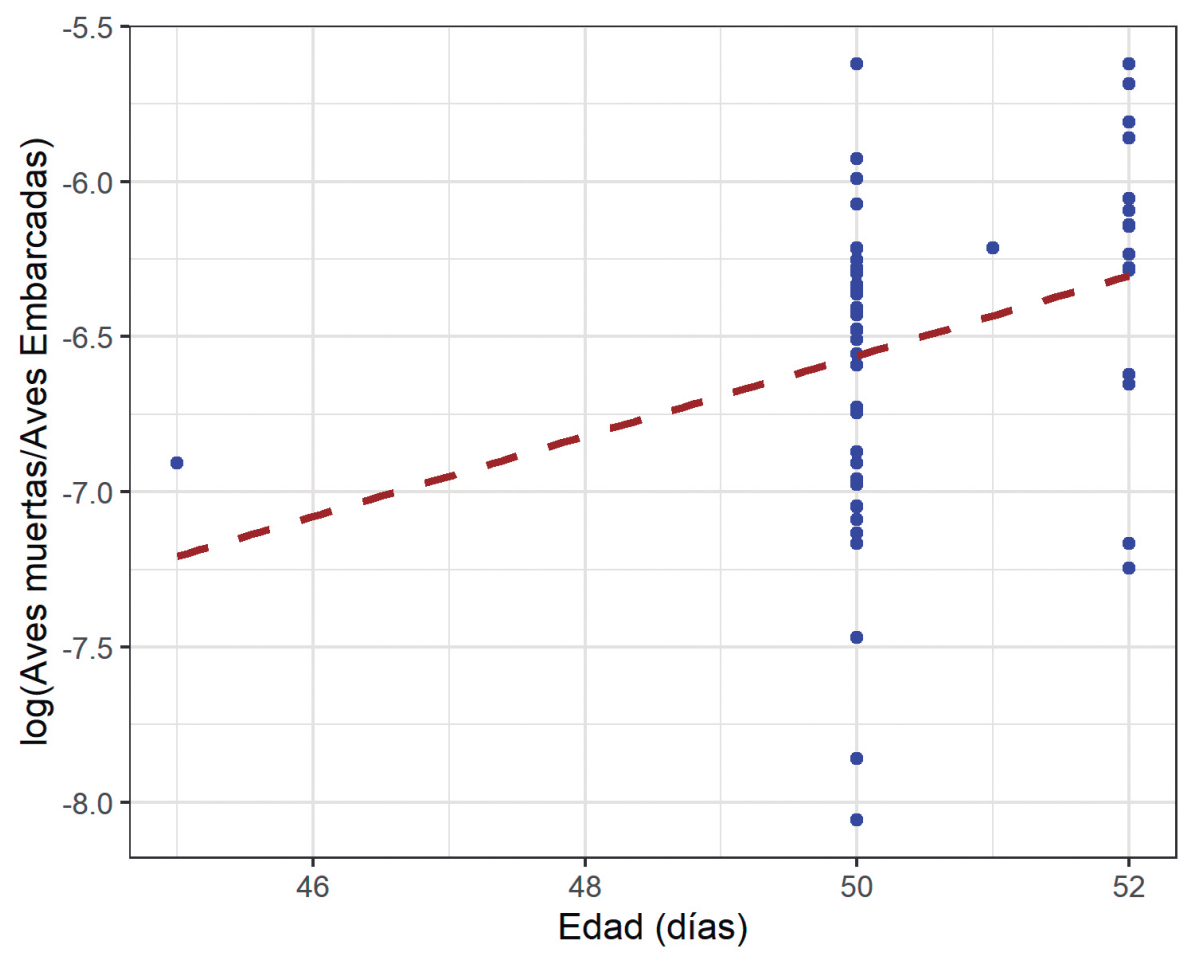

Figura 1. Efecto de la edad al momento del faenamiento sobre la mortalidad de pollos.

Así mismo, la temperatura de embarque acusó un efecto asociado sobre la mortalidad de los pollos, evidenciando un 3,5\% ( $\mathrm{IC}_{95 \%}: 0,01 \%$ $-7,0 \%)$ de incremento en la mortalidad por cada grado de descenso en la temperatura ambiental a la hora del embarque de pollos (Figura 2), siendo estas dos variables las que permanecieron en el modelo final reducido.

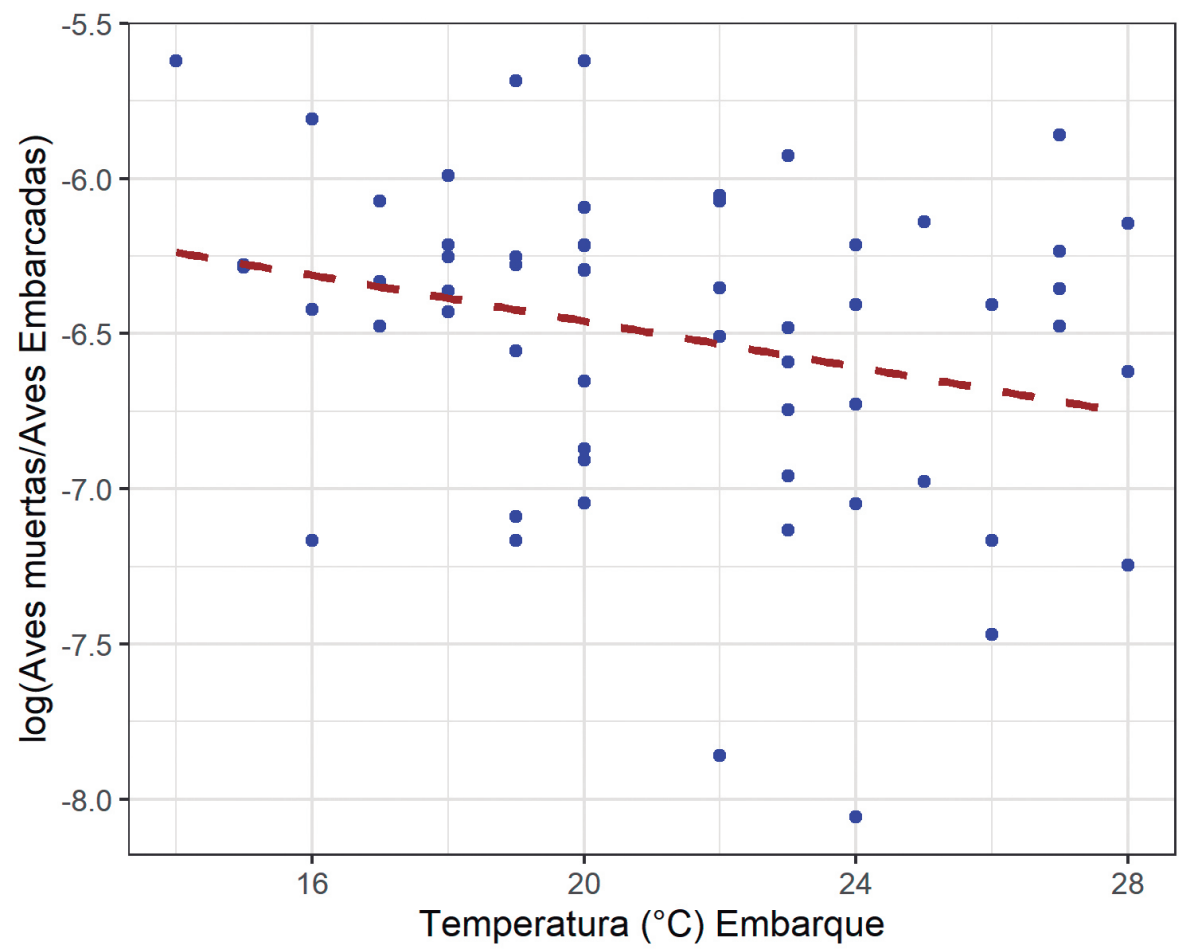

Figura 2. Efecto de las variaciones de temperatura de embarque sobre la mortalidad durante el transporte de pollos. 
La Figura 3 expresa la interrelación de las variables edad y temperatura al embarque, con la mor- talidad de los pollos, las cuales explican un efecto simultáneo.

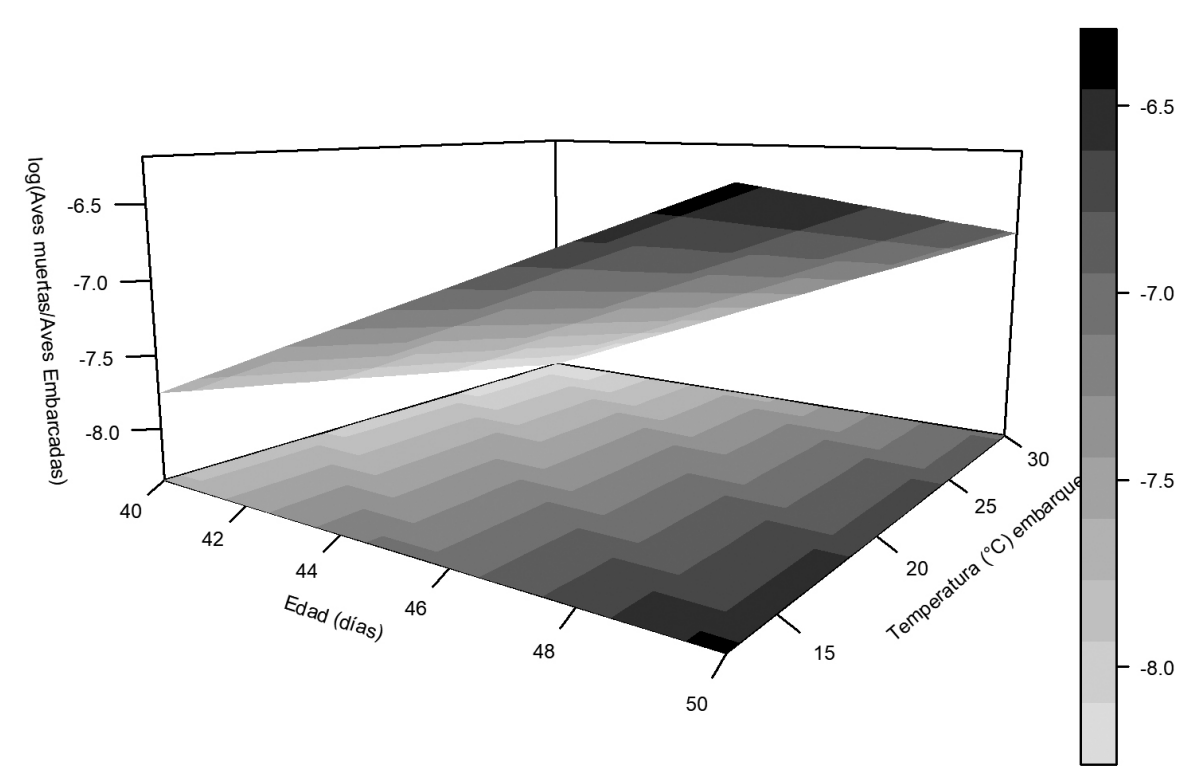

Figura 3. Efecto combinado de la edad a la faena y la temperatura de embarque sobre la mortalidad $(\mathrm{Rt}=\log$ mortalidad/pollos embarcados) de pollos de engorde.

\section{Discusión}

Las variables sexo de los pollos, capacidad de las jaulas, línea genética, tiempo de viaje, kilómetros recorridos, y velocidad de viaje no influyeron significativamente sobre la variable de respuesta. Es probable que las distancias relativamente cortas registradas en el estudio, el buen estado de vías, y particularmente las prácticas de bienestar en jaulas de transporte, las cuales no superaron 8 pollos por jaula, expliquen estos resultados. Al respecto, Fernández (2003) asegura que el transporte tanto en trayectos cortos como en viajes de larga duración, causa estrés en los animales, aunque afirman que lo importante no es el tiempo de transporte sino las condiciones en las que se realice, debido a que podría ocasionar pérdidas de peso en los animales transportados.

El sexo en este estudio no acusó relación con la mortalidad de pollos, lo cual contrasta con otros estudio como los reportados por Vargas et al. (2005) y Whiting et al. (2007) quienes manifiestan que existiría una relación significativa entre las mermas por transporte de machos y la mortalidad en el trayecto, es decir, el menor riesgo de mortalidad en cargas que contienen solo pollos hembras en comparación con las que contienen machos podría deberse a una mayor capacidad de las hembras para mantener su temperatura corporal, en razón de su mayor cobertura de plumas y grasa abdominal, pero menor peso corporal; cuando se exponen a temperaturas frías (por ejemplo, $8^{\circ} \mathrm{C}$ ) (Dadgar et al., 2011).

En relación con la variable línea genética, ciertos estudios demuestran diferencias para las líneas Cobb y Ross que representan respuestas diferenciadas frente al comportamiento de cada línea en las granjas en cuanto a los estímulos de estrés horas antes del sacrificio (Nunes, 1998; Spíner, 2002). Otros estudios epidemiológicos han identificado y señalado múltiples factores de riesgo de mortalidad de aves durante el transporte, siendo la raza o línea genética, el tamaño del lote, peso vivo, la tasa de mortalidad durante el período de cría, el método de captura, la hora del día, densidad de carga de las cajas, condiciones climáticas, duración del viaje, duración de la carga y estrés térmico, son algunos ejemplos (Nijdam et al., 2004, Whiting et al., 2007; Knezacek et al., 2010; Alshawabkeh y Tabbaa, 1997; Dam y Fitzgerald, 2017; Barbosa Filho et al., 2014).

La mortalidad de pollos en este estudio fue de 1,6 por cada mil pollos, es decir, 213 pollos muertos de una población de 130.467 pollos embarcados desde la granja hasta la planta de faenamiento. Estos hallazgos demuestran una baja tasa de mortalidad de pollos, que se explica por el poco tiempo de recorrido, las distancias cortas y a una baja velocidad de 
transporte en vías de primer orden, que en promedio fue $2,67 \pm 1,56$ horas, $123,28 \pm 92,91 \mathrm{~km}$ y $44,21 \pm$ $12,63 \mathrm{~km} /$ hora, respectivamente. A pesar de ello, no se descarta que varios animales sufrieran de hipotermia y/o hipertermia durante el transporte por efecto de la densidad de las cajas en el camión, que obligaría a que los pollos respondan en términos fisiológicos y conductuales. Al respecto, Francia et al. (2009) y Bayliss y Hinton (1990) afirman que los pollos sometidos a cortos periodos de estrés en el transporte, se exponen menos y, por lo tanto, la mortalidad es mínima asociada a la distancia entre galpón-planta de faenamiento. Otros autores coinciden en afirmar que las condiciones climáticas de frío o calor que experimentan en las cajas de la plataforma del camión, serían causas importantes de mortalidad de pollos (Figueroa Fuentes, 1997; Caffrey et al., 2017; dos Santos et al., 2017).

Los pollos con más de 50 días de edad al momento del embarque, y temperatura de embarque inferiores a $21,25^{\circ} \mathrm{C}$, reflejaron correlaciones significativas con la mortalidad de pollos. El análisis de la Tabla 3 y Figura 2, muestra que la edad de los po1 los al momento de la captura fue un factor causal de incremento de $17 \%$ extra en la mortalidad por cada día adicional de permanencia de los pollos en el galpón. Este hecho podría atribuirse a que las aves de mayor edad, son más pesadas y acusan mayor riesgo de mortalidad, tal como sugieren otros estudios que afirman que los factores de las aves: el sexo, la mayor edad y el incremento de peso, aumentaba el riesgo de mortalidad (Teke, 2019). Además, el peso corporal más ligero de las hembras en comparación con los machos, podría tornarles menos propensas a la muerte por estrés por calor (Chauvin et al., 2011; Nijdam et al., 2004; Whiting et al., 2007; Haslam et al., 2008). Contrariamente, otros estudios presumen que el riesgo de mortalidad aumenta en las aves más jóvenes (33-37 días de edad) de ambos sexos en comparación con las de 38 a 39 días de edad, lo cual podría estar relacionado con el aumento de la capacidad de los pollos de engorde de 40 a 42 días de edad (con mayor tamaño corporal y mayor cobertura de plumas) en comparación con los 35-37 días de edad para mantener su temperatura corporal cuando se exponen a temperaturas frías (Dadgar et al., 2011).

De hecho, el ayuno comprende dos etapas fundamentales, las cuales comienzan en la granja al momento de retirar el alimento al levantar los comederos y se prolonga hasta el transporte de las aves; tal como mencionan algunos autores (Nunes, 2001; Hincapié y Rodas, 2001; Cervantes, 2000). Una vez que son retirados los equipos dentro del galpón, se priva de alimento a las aves con el fin de disminuir el contenido digestivo (Hincapié y Rodas, 2001) para que al cabo de unas cuantas horas los animales sean capturados y trasladados en camiones hasta la planta de sacrificio. Nilipour (2001) recomienda que este tiempo no debe ser mayor de una hora para evitar pérdidas de peso y mortalidades en los pollos. En términos promedios, en este estudio los pollos permanecieron alrededor de 8 horas en ayuno.

De igual manera, la temperatura de embarque causó un incremento adicional del 3,5\% de la mortalidad por cada grado de descenso en la temperatura ambiental a la hora del embarque de pollos, respecto de la mortalidad base. Estos resultados sugieren que al momento de la captura antes del embarque, aparentemente se produce un cambio brusco de la temperatura corporal de una proporción de la población de pollos, provocando una alteración en su capacidad termorreguladora. El estrés térmico, es decir, los cambios ambientales repentinos durante la carga, el tipo de transporte y las prácticas de descarga en sitio de faenamiento (Mitchell y Kettlewell, 2009; Vecerek et al., 2006; Vecerek et al., 2016), causan lesiones físicas (Whiting et al., 2007) durante la captura y la carga que explican las mermas de pollos. Cuando las características del viaje son tales que se superan las capacidades termorreguladoras de las aves para la homeotermia, el riesgo de la mortalidad para la carga aumenta y el porcentaje de aves que mueren debido al estrés térmico también aumenta (Hunter et al., 2001; dos Santos et al., 2017; Wang et al., 2013).

En la Figura 3, se puede apreciar el efecto combinado de la edad y temperatura de embarque sobre la mortalidad de pollos. Eso significa que, los pollos de mayor edad al sacrificio y temperaturas menores al promedio de $22,5^{\circ} \mathrm{C}$ al momento del embarque, la mortalidad aumenta, aunque la proporción relativa de pollos que mueren a causa de estos dos factores en conjunto, podría alterarse según las variaciones ambientales que experimentan los pollos durante el viaje. Así, cuando las características del viaje exceden la capacidad termorreguladora de los pollos para homeotermia, aumenta el riesgo de mortalidad ya sea por la carga, o por estrés térmico (Hunter et al., 2001; Tolentino et al., 2008). De hecho, la temperatura promedio en lugar de destino, fue en promedio de $13,5{ }^{\circ} \mathrm{C}$, en cuyo caso, los pollos pudieron sufrir de hipotermia (Warriss et al., 2005).

De otra parte, según Burlinguette et al. (2012) las condiciones térmicas al interior de un camión que 
contiene pollos de engorde, no siempre son adecuadas, dado que la mayoría de los pollos de engorde se transportan en contenedores (cajas o módulos) apilados en un remolque de plataforma que está abierto total o parcialmente en los laterales y cerrado con paneles sólidos en los lados frontal, posterior y superior, a menudo con aberturas ajustables para permitir la entrada y salida de aire (Knezacek et al., 2010). En este sentido, esta investigación estimula a realiza otros estudios para medir el efecto de los cambios térmicos durante la transportación de las aves con la finalidad de bajar el riesgo de mermas en los pollos que se destinan al mercado.

Una vez en la planta de faenamiento, cuando los pollos de engorde se descargan de sus cajas, los animales muertos se describen como "muertos a la llegada" o DOA (Dead on Arrival, por sus siglas en inglés). Sin embargo, esta mortalidad puede haber ocurrido en cualquier momento después de la carga en la granja, durante el viaje, entre otras causas. Estas aves muertas son consideradas no aptas para el consumo humano, representando una pérdida económica. El riesgo de mortalidad o el porcentaje de DOA en una carga de pollos proporciona una indicación de la gravedad de los problemas de bienestar animal experimentados por los animales (Jacobs et al., 2017; Warriss, 2005; Caffrey et al., 2017; Caffrey et al., 2017; Ristic y Damme, 2013; Schwartzkopf-Genswein et al., 2012).

Por lo expuesto, varios autores coinciden en señalar que el transporte debe realizarse bajo pará-

\section{Referencias}

Adzitey, F., \& Nurul, H. (2011). Pale Soft Exudative(PSE) and Dark Firm Dry (DFD) meats: Causes and measures to reduce these incidences. A mini review. International Food Research Journal, 18, 11-17.

Alshawabkeh, K., \& Tabbaa, M. J. (1997). Factors affecting mortality and losses during transportation of broiler chickens from farms to processing plants in Jordan. Irasat Agricultural Sciences, 24, 53-61.

Arbor Acres. (2009). Guía de manejo del pollo de engorde. Aviagen Incorporated \& Aviagen Limited. metros de legislación local, previo a la llegada a la sala de faenamiento, los pollos deben estar protegidos de las distintas condiciones medioambientales, proporcionado ventilación, calefacción y cuando sea necesario, enfriamiento parcial (Aviagen, 2014; Prado et al., 2010; Pita Ponte, 2015; Wichman et al., 2012) y otras medidas como poner límites a factores como la densidad de carga de pollos de engorde, además de aplicar planes voluntarios de certificación que pueden lograr mayores niveles de bienestar (FAO, 2012). Este estudio permite señalar que, a edades más juveniles, los pollos sufren menos estrés por transporte y, por tanto, acusan menor mortalidad.

\section{Conclusiones}

En las condiciones en que se realizó este estudio, la edad de los pollos al sacrificio y temperatura al embarque, fueron los factores de riesgo que influyeron sobre la mortalidad de pollos, así como sobre el bienestar animal, debido a la exposición de los pollos a una condición de estrés térmico que se agravó con la edad. El sexo, capacidad de las jaulas, línea genética, tiempo de viaje, distancia recorrida, velocidad de viaje, humedad relativa de desembarque; no demostraron una relación con la mortalidad de aves. Los resultados podrían utilizarse para perfeccionar las prácticas de manejo a fin de mitigar parte del riesgo de mortalidad de los pollos durante el transporte desde las granjas hasta el lugar de faenamiento.

Aviagen (2014). Manual de manejo del pollo de engorde Ross. Aviagen Incorporated.

Barbosa Filho, J. A. D., Queiroz, Marília L. V., Brasil, D. de F., Vieira, F. M. C., \& Silva, I. J. O. (2014). Transport of broilers: load microclimate during Brazilian summer. Engenharia Agrícola, 34(3), 405-412. https://doi. org/10.1590/S0100-69162014000300003

Barbut, S. (2015). The science of poultry and meat processing. University of Guelph, Food Science Department. http://www.poultryandmeatprocessing.com/ 
Bayliss, P. A., \& Hinton, M. H. (1990). Transportation of broilers with special reference to mortality rates. Applied Animal Behaviour Science, 28(1-2), 93-118. https://doi.org/10.1016/0168-1591(90)90048-I

Burlinguette, N. A., Strawford, M. L., Watts, J. M., Classen, H. L., Shand, P. J., \& Crowe, T. G. (2012). Broiler trailer thermal conditions during cold climate transport. Canadian Journal of Animal Science, 92(2), 109-122. https://doi. org/10.4141/cjas2011-027

Caffrey, N. P., Dohoo, I. R., \& Cockram, M. S. (2017). Factors affecting mortality risk during transportation of broiler chickens for slaughter in Atlantic Canada. Preventive veterinary medicine, 147, 199-208. https://doi.org/10.1016/j. prevetmed.2017.09.011

Cervantes, E. (2000). Procesamiento de aves: actividad donde hay que cuidar los gramos. Industria Avicola, 24-27.

Chauvin, C., Hillion, S., Balaine, L., Michel, V., Peraste, J., Petetin, I., Lupo, C., \& Le Bouquin, S. (2011). Factors associated with mortality of broilers during transport to slaughterhouse. Animal: an international journal of animal bioscience, 5(2), 287-293. https://doi.org/10.1017/ S1751731110001916

Corporacion Nacional de Avicultores del Ecuador [Conave]. (2021). Estadísticas avícolas. Obtenido de https://www.conave.org/informacion-sector-avicola-publico/

Cuadros Colmenares, C. (2006). Evaluación de la merma de pollo de engorde durante el transporte de la granja hasta el inicio del proceso de beneficio para Coopvencedor. Universidad de La Salle.

Dadgar, S., Lee, E. S., Leer, T. L. V., Crowe, T. G., Classen, H. L., \& Shand, P. J. (2011). Effect of acute cold exposure, age, sex, and lairage on broiler breast meat quality. Poultry Science, 90(2), 444-457. https://doi.org/10.3382/ ps.2010-00840

Dam, A., \& Fitzgerald, S. (Eds.) (2017). Poultry Handling and Transportation Manual. Ministry of Agriculture, Food and Rural Affairs, \& Poultry Service Association. dos Santos, V. M., Dallago, B. S., Racanicci, A. M., Santana, A. P., \& Bernal, F. E. (2017). Effects of season and distance during transport on broiler chicken meat. Poultry science, 96(12), 4270-4279. https://doi.org/10.1371/journal. pone. 0232004

FAO. (2012). Aves de corral y el bienestar. Organización de las Naciones Unidas para la Alimentación y la Agricultura, Departamento de Agricultura y Protección al Consumidor. Obtenido de http://www.fao.org/Ag/againfo/themes/es/ poultry/AH_welfare.html

Fernández, M. (2003). Los efectos del transporte sobre el ganado bovino. Obtenido de https:// www.consumer.es/seguridad-alimentaria/ los-efectos-del-transporte-sobre-el-ganado-bovino.html

Figueroa Fuentes, A. (1997). Evaluación de la merma de peso del pollo de engorde desde el transporte, hasta su sacrificio en la planta de proceso. Escuela Agrícola Panamericana, Zamorano.

Francia, M., Icochea, E., Reyna, P., \& Figueroa, E. (2009). Tasas de mortalidad, eliminados, y descartes de dos líneas genéticas de pollos de engorde. Revista de Investigaciones Veterinarias de Perú, 20(2), 228-234. https://doi. org/10.15381/rivep.v20i2.612

Grilli, C., Loschi, A. R., Rea, S., Stocchi, R., Leoni, L., \& Conti, F. (2015). Welfare indicators during broiler slaughtering. British poultry science, 56(1), 1-5. https://doi.org/10.1080/0007166 8.2014 .991274

Habibu, B., Ikira, N.M., Buhari, H., Aluwong, T., Kawu, M., Yaqub, L.S., Tauheed, M., \& Isa, H.I. (2014). Effect of molasses supplementation on live weight gain, haematologic parameters and erythrocyte osmotic fragility of broiler chickens in the hot-dry season. International Journal of Veterinary Science, 3(4), 181-188.

Haslam, S. M., Knowles, T. G., Brown, S. N., Wilkins, L. J., Kestin, S. C., Warriss, P. D., \& Nicol, C. J. (2008). Prevalence and factors associated with it, of birds dead on arrival at the slaughterhouse and other rejection conditions in broiler chickens. British poultry science, 49(6), 685-696. https://doi.org/10.1080/00071660802433719 
Hincapié, J. J., \& Rodas, R. E. (2001). Cría y explotación del pollo de engorde. Manual técnico. Ed. Zamorano.

Hunter, R. R., Mitchell, M. A., \& Matheu, C. (2001). Mortality of broiler chickens in transit - correlation with the thermal micro-environment. In: Livestock Environment VI: Proceedings of the 6th International Symposium (pp. 542-549). Louisville, Kentucky, USA.

Jacobs, L., Delezie, E., Duchateau, L., Goethals, K., \& Tuyttens, F. A. (2017). Broiler chickens dead on arrival: associated risk factors and welfare indicators. Poultry science, 96(2), 259-265. https://doi.org/10.3382/ps/pew353

Knezacek, T. D., Olkowski, A. A., Kettlewell, P. J., Mitchell, M. A., \& Classen, H. L. (2010). Temperature gradients in trailers and changes in broiler rectal and core body temperature during winter transportation in Saskatchewan. Canadian Journal of Animal Science, 90(3), 321330. https://doi.org/10.4141/CJAS09083

Mitchell, M. A., \& Kettlewell, P. J. (1998). Physiological stress and welfare of broiler chickens in transit: solutions not problems!. Poultry science, 77(12), 1803-1814. https://doi.org/10.1093/ ps/77.12.1803

Mitchell, M.A., \& Kettlewell, P.J. (2009). Welfare of poultry during transport - a review. In Proceedings of the 8th European Symposium on Poultry Welfare (pp. 90-100). Cervia, Italy.

Moreno Vásquez, F. C., \& Chinchilla Suárez, M. R. (2007). Análisis del efecto de la temperatura y la humedad relativa sobre el consumo de alimento y aumento del peso corporal en un sistema de producción de pollo de engorde en el Municipio de Arbeláez (Cundinamarca). Revista Colombiana de Ciencias Pecuarias, 20(4), 566.

Nijdam, E., Arens, P., Lambooij, E., Decuypere, E., \& Stegeman, J. A. (2004). Factors influencing bruises and mortality of broilers during catching, transport, and lairage. Poultry science, 83(9), 1610-1615. https://doi.org/10.1093/ ps/83.9.1610

Nilipour, A. (2001). Reduciendo la mortalidad en recepción en la planta de proceso. Industria Avícola, 22.
Nunes, F. (1998). Cuidado desde la granja hasta la planta. Avicultura Profesional, 30-35.

Nunes, F. (2001). El mercado venezolano de un vistazo. Industria Avícola, 8.

Pita Ponte, E. (2015). Bienestar en el transporte de aves. El Sitio Avicola. Obtenido de http://www. elsitioavicola.com/articles/2786/bienestar-enel-transporte-de-aves/

Prado, O., Morales, E., \& Macedo, R. (2010). Factores de riesgo relacionados con tiempo de retiro de agua y alimento, captura, transporte y espera sobre la mortalidad de pollos. En Memorias de la Tercera Reunión Anual de la Asociación de Especialistas en Ciencias Avícolas del Centro de México A.C. (pp. 86-92). Asociación de Especialistas en Ciencias Avícolas del Centro de México A.C.

Ristic, M., \& Damme, K. (2013). Significance of $\mathrm{pH}$-value for meat quality of broilers: Influence of breed lines. Veterinarski glasnik, 67(1/2), 6773. https://doi.org/10.2298/VETGL1302067R

Ruiz, B. (2017). Producción avícola a nivel nacional. Industria Avícola, 64(3), 18-19.

Schwartzkopf-Genswein, K. S., Faucitano, L., Dadgar, S., Shand, P., González, L. A., \& Crowe, T. G. (2012). Road transport of cattle, swine and poultry in North America and its impact on animal welfare, carcass and meat quality: a review. Meat science, 92(3), 227-243. https:// doi.org/10.1016/j.meatsci.2012.04.010

Spiner, N., \& Brunori, J. (2002). Transporte de cerdos al mercado: recomendaciones para disminuir las pérdidas de cerdos por el mal manejo de la carga y el transporte. EEA INTA Marcos Juárez.

Teke, B. (2019). Survey on dead on arrival of broiler chickens under commercial transport conditions. Large Animal Review, 25, 237-241.

Tolentino, C., Icochea, E., Reyna, P., \& Valdivia, R. (2008). Influencia de la temperatura y humedad ambiental del verano e invierno sobre parámetros productivos de pollos de carne criados en la ciudad de Lima. Revista de Investigaciones Veterinarias del Perú, 19(1), 9-14. https://doi. org/10.15381/rivep.v19i1.1169 
Vargas, M. R., Moreno Vàsquez, F. C., \& Forero, E. A. (2005). Evaluación del efecto del tiempo de transporte sobre la pérdida de peso de pollos de engorde en dos líneas comerciales. Revista de Medicina Veterinaria, 10, 78-94.

Vecerek, V., Grbalova, S., Voslarova, E., Janackova, B., \& Malena, M. (2006). Effects of travel distance and the season of the year on death rates of broilers transported to poultry processing plants. Poultry science, 85(11), 1881-1884. https://doi.org/10.1093/ps/85.11.1881

Vecerek, V., Voslarova, E., Conte, F., Vecerkova, L., \& Bedanova, I. (2016). Negative Trends in Transport-related Mortality Rates in Broiler Chickens. Asian-Australasian Journal of Animal Sciences, 29(12), 1796-1804. https://doi. org/10.5713/ajas.15.0996

Vecerkova, L., Vecerek, V., \& Voslarova, E. (2019). Welfare of end-of-lay hens transported for slaughter: effects of ambient temperature, season, and transport distance on transport-related mortality. Poultry science, 98(12), 6217-6224. https://doi.org/10.3382/ps/pez468

Villarroel, M., Pomares, F., Ibáñez, M., Lage, A., Martínez-Guijarro, P., Méndez, J., \& de Blas, C. (2018). Rearing, bird type and pre-slaughter transport conditions I. Effect on dead on arrival. Spanish Journal of Agricultural Research, 16(2), e0503. http:// dx.doi.org/10.5424/sjar/2018162-12013

Wang, Y., Jiang, Z., Jin, Z., Tan, H., \& Xu, B. (2013). Risk factors for infectious diseases in backyard poultry farms in the Poyang Lake area, China. PloS one, 8(6), e67366. https://doi.org/10.1371/ journal.pone. 0067366

Warriss, P. D., Pagazaurtundua, A., \& Brown, S. N. (2005). Relationship between maximum daily temperature and mortality of broiler chickens during transport and lairage. British poultry science, 46(6), 647-651. https://doi. org/10.1080/00071660500393868

Webster, A. J., Tuddenham, A., Saville, C. A., \& Scott, G. B. (1993). Thermal stress on chickens in transit. British poultry science, 34(2), 267-277. https://doi.org/10.1080/00071669308417583

Weeks, C. A., Brown, S. N., Richards, G. J., Wilkins, L. J., \& Knowles, T. G. (2012). Levels of mortality in hens by end of lay on farm and in transit to slaughter in Great Britain. The Veterinary record, 170(25), 647. https://doi.org/10.1136/vr.100728

Whiting, T. L., Drain, M. E., \& Rasali, D. P. (2007). Warm weather transport of broiler chickens in Manitoba. II. Truck management factors associated with death loss in transit to slaughter. The Canadian veterinary journal $=L$ a revue veterinaire canadienne, 48(2), 148-154.

Wichman, A., Norring, M., Voutila, L., Pastell, M., Valros, A., Algers, B., \& Hänninen, L. (2012). Influence of crate height during slaughter transport on the welfare of male turkeys. British poultry science, 53(4), 414-420. https://doi.org /10.1080/00071668.2012.711465 